\title{
Solving Variational Inequalities Defined on A Domain with Infinitely Many Linear Constraints
}

\author{
Shu-Cherng Fang, Soonyi Wu, Ş. İlker Birbil
}

\begin{tabular}{|l|l|}
\hline \multicolumn{2}{|l|}{ ERIM REPORT SERIES RESEARCH IN MANAGEMENT } \\
\hline ERIM Report Series reference number & ERS-2002-70-LIS \\
\hline Publication & July 2002 \\
\hline Number of pages & 16 \\
\hline Email address corresponding author & sibirbil@few.eur.nl \\
\hline Address & Erasmus Research Institute of Management (ERIM) \\
& Rotterdam School of Management / Faculteit Bedrifskunde \\
& Erasmus Universiteit Rotterdam \\
& P.O. Box 1738 \\
& 3000 DR Rotterdam, The Netherlands \\
& Phone: +31 10 408 1182 \\
& Fax: $\quad+31104089640$ \\
& Email: info@erim.eur.nl \\
& Internet: $\quad$ www.erim.eur.nl \\
\hline
\end{tabular}

Bibliographic data and classifications of all the ERIM reports are also available on the ERIM website: www.erim.eur.nl 
REPORT SERIES

RESEARCH IN MANAGEMENT

\begin{tabular}{|c|c|c|}
\hline \multicolumn{3}{|c|}{ BIBLIOGRAPHIC DATA AND CLASSIFICATIONS } \\
\hline Abstract & \multicolumn{2}{|c|}{$\begin{array}{l}\text { We study a variational inequality problem whose domain is defined by infinitely many linear } \\
\text { inequalities. A discretization method and an analytic center based inexact cutting plane method } \\
\text { are proposed. Under proper assumptions, the convergence results for both methods are } \\
\text { given. We also provide numerical examples for the proposed methods. }\end{array}$} \\
\hline \multirow{3}{*}{$\begin{array}{l}\text { Library of Congress } \\
\text { Classification } \\
\text { (LCC) }\end{array}$} & $5001-6182$ & Business \\
\hline & $5201-5982$ & Business Science \\
\hline & QA 299.6-433 & Analysis, including analytical methods \\
\hline \multirow{4}{*}{$\begin{array}{l}\text { Journal of Economic } \\
\text { Literature } \\
\text { (JEL) }\end{array}$} & M & Business Administration and Business Economics \\
\hline & M 11 & Production Management \\
\hline & R 4 & Transportation Systems \\
\hline & C 69 & Mathematical methods: other \\
\hline \multirow{4}{*}{$\begin{array}{l}\text { European Business Schools } \\
\text { Library Group } \\
\text { (EBSLG) }\end{array}$} & $85 \mathrm{~A}$ & Business General \\
\hline & $260 \mathrm{~K}$ & Logistics \\
\hline & $240 \mathrm{~B}$ & Information Systems Management \\
\hline & $250 \mathrm{~A}$ & Mathematics \\
\hline \multicolumn{3}{|c|}{ Gemeenschappelijke Onderwerpsontsluiting (GOO) } \\
\hline \multirow[t]{4}{*}{ Classification GOO } & 85.00 & Bedrijfskunde, Organisatiekunde: algemeen \\
\hline & 85.34 & Logistiek management \\
\hline & 85.20 & Bestuurlijke informatie, informatieverzorging \\
\hline & 31.48 & Variatierekening \\
\hline \multirow[t]{3}{*}{ Keywords GOO } & \multicolumn{2}{|c|}{ Bedrijfskunde / Bedrijfseconomie } \\
\hline & \multicolumn{2}{|c|}{ Bedrijfsprocessen, logistiek, management informatiesystemen } \\
\hline & \multicolumn{2}{|c|}{ Variatieongelijkheden } \\
\hline Free keywords & \multicolumn{2}{|c|}{$\begin{array}{l}\text { Variational inequality problem, analytic center, cutting plane method, discretization method, } \\
\text { inexact approach }\end{array}$} \\
\hline
\end{tabular}




\title{
Solving Variational Inequalities Defined on A Domain with Infinitely Many Linear Constraints
}

\author{
Shu-Cherng Fang * Soonyi Wu ${ }^{\dagger \S} \quad$ Ş. İlker Birbil ${ }^{\ddagger}$
}

July 24, 2002

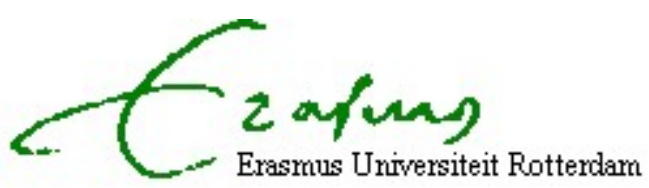

\begin{abstract}
We study a variational inequality problem whose domain is defined by infinitely many linear inequalities. A discretization method and an analytic center based inexact cutting plane method are proposed. Under proper assumptions, the convergence results for both methods are given. We also provide numerical examples for the proposed methods.
\end{abstract}

Keywords Variational inequality problem, analytic center, cutting plane method, discretization method, inexact approach.

\footnotetext{
* Industrial Engineering and Operations Research, North Carolina State University, Raleigh, NC 26695-7906, USA. E-mail: fang@eos.ncsu.edu

${ }^{\dagger}$ Institute of Applied Mathematics, National Cheng Kung University, Tainan, Taiwan 700, R.O.C. E-mail: soonyi@mail.ncku.edu.tw

${ }^{\ddagger}$ Erasmus Research Institute of Management (ERIM), Erasmus University, Postbus 1738, 3000 DR Rotterdam, The Netherlands. E-mail: sibirbilefew.eur.nl

$\S$ This author's work was partially supported by the National Science Council, Taiwan, R.O.C.(Grant No. 19731001)
} 


\section{Introduction}

Let $X$ be a nonempty subset of $R^{n}$ and $F$ be a function from $R^{n}$ to itself. The finite dimensional variational inequality problem, denoted by $\mathrm{VI}(X, F)$, is to find a vector $x^{*} \in X$ such that

$$
F\left(x^{*}\right)^{T}\left(x-x^{*}\right) \geq 0 \text { for all } x \in X .
$$

The development of the theory, algorithms and applications of finite dimensional variational inequalities can be found in [Harker and Pang, 1990]. Although the theory is very rich, the history of designing algorithms for finite dimensional variational inequalities is relatively short. Moreover in practice, most algorithms work only when $X$ exhibits a certain special geometric structure (such as the positive orthant of $R^{n}$ or a polyhedral set).

Like in [S.-C. Fang and Sun, 2002], in this paper we consider a setting for which $X$ is a nonempty, bounded set defined by

$$
X=\left\{x \in R^{n} \mid\langle u(t), x\rangle-\lambda(t) \leq 0 \text { for all } t \in T\right\},
$$

where $T$ is a nonempty compact subset of $R, u(t): T \rightarrow R^{n}$ and $\lambda(t): T \rightarrow R$ are continuous on $T$. Since there may be infinitely many linear inequalities involved in defining $X$, we call this setting a linear semi-infinite variational inequality problem, or $\operatorname{LSIVI}(X, F)$ in short.

Notice that when $F$ is a continuous pseudomonotone mapping (to be defined in later sections) from $X$ to $R^{n}$, it is not difficult to prove that $x^{*} \in X$ solves $\operatorname{LSIVI}(X, F)$ if and only if it solves the following problem:

$$
F(x)^{T}\left(x-x^{*}\right) \geq 0 \text { for all } x \in X,
$$

which we denote as $\operatorname{DLSIVI}(X, F)$. Also notice that $\operatorname{DLSIVI}(X, F)$ is equivalent to a convex feasibility problem [J.-L. Goffin and Ye, 1993, 1996, J.-L. Goffin and Zhu, 1997, Goffin and Vial, 1999, Luo and Sun, 1999, 2000], i.e., finding a point $x^{*}$ in a convex set defined by an infinite number of linear inequalities

$$
S=\left\{x^{*} \in X \mid F(x)^{T} x^{*} \leq F(x)^{T} x \text { for all } x \in X\right\} .
$$

A new development is using analytic center based cutting plane methods to solve variational inequalities [J.-L. Goffin and Zhu, 1997, Lüthi and Bueler, 2000, Magnanti and Perakis, 1995, Marcotte and Zhu, 2001]. This approach combines the feature of interior point methods with the classical cutting plane scheme. Recently, the authors in [S.-C. Fang and Sun, 2002] presented an analytic center based cutting plane method for solving a general semi-infinite variational inequality problem.

In this paper, we focus on the semi-infinite variational inequality problems whose domains are defined by infinitely many linear constraints. We first study a discretization approach for solving $\operatorname{LSIVI}(X, F)$ and show a convergence result under proper assumptions. The quality of solutions obtained by the discretization approach depends on the expansive sequence used. It is hard to provide any quantitative statement. Then, we propose an analytic center based "inexact" cutting plane method and give its convergence proof. Unlike other cutting plane methods, such as the one used in [S.-C. Fang and Sun, 2002], the proposed method requires only an "inexact" solution to a variational inequality problem at each iteration. Also, the quality of solutions obtained by the proposed inexact method can be carefully analyzed. 
This paper is organized as follows. Some preliminaries are given in Section 2. We discuss the discretization method for $\operatorname{LSIVI}(X, F)$ and show a convergence result in Section 3. An analytic center based inexact cutting plane method is proposed with a convergence proof in Section 4. The computational results over a set of problems are reported in Section 5. The paper is concluded in Section 6.

\section{Preliminaries}

Since an analytic center is usually defined at an interior point of a given region, we make the following "interior point" assumption throughout this paper:

There exists an $\hat{x} \in R^{n}$ such that

$$
\langle u(t), \hat{x}\rangle-\lambda(t)<0 \text { for all } t \in T .
$$

The interior point assumption assures that $X$ has a nonempty interior. It is easy to see that $X$ is a convex set. Moreover, the continuity of $\langle u(t), x\rangle-\lambda(t)$ on $R^{n} \times T$ implies that $X$ is a closed set. Remember that in our setting, $X$ is assumed to be bounded. Consequently, $X$ is a nonempty, convex, and compact subset of $R^{n}$ and the next result follows:

Proposition 1 [S.-C. Fang and Sun, 2002] Under the interior point assumption, if $F$ is a continuous mapping from $X$ to $R^{n}$, then there exists a solution to $\operatorname{LSIVI}(X, F)$.

Let us recall some definitions on the mappings commonly used for a variational inequality problem $\mathrm{VI}(X, F)$.

Definition 1 [J.-L. Goffin and Zhu, 1997, Marcotte and Zhu, 2001] A mapping $F$ is said to be

- monotone on $X$ if for every pair of points $x, y \in X$,

$$
(F(x)-F(y))^{T}(x-y) \geq 0 .
$$

- strongly monotone on $X$ if there exists $\beta>0$ such that for every pair of points $x, y \in X$,

$$
(F(x)-F(y))^{T}(x-y) \geq \beta\|x-y\|^{2} .
$$

- pseudomonotone on $X$ if for every pair of points $x, y \in X$,

$$
F(x)^{T}(y-x) \geq 0 \text { implies } F(y)^{T}(y-x) \geq 0 .
$$

- pseudomonotone-plus on $X$ if it is pseudomonotone on $X$ and for every pair of points

$$
F(x)^{T}(y-x)=0 \text { and } F(y)^{T}(y-x)=0 \text { imply } F(x)=F(y) .
$$

- pseudo-co-coercive with modulus $\alpha>0$ on $X$ if for every pair of points $x, y \in X$,

$$
F(x)^{T}(y-x) \geq 0 \text { implies } F(y)^{T}(y-x) \geq \alpha\|F(x)-F(y)\|^{2} .
$$

It is not difficult to see that a monotone mapping is pseudomonotone and a strongly monotone mapping is pseudo-co-coercive. Moreover, the following result can be found in [S.-C. Fang and Sun, 2002]. 
Proposition 2 Let $F$ be a continuous pseudomonotone mapping over $X$. Under the interior point assumption, $x^{*} \in X$ is a solution to $\operatorname{LSIVI}(X, F)$ if and only if $x^{*}$ solves

$\operatorname{DLSIVI}(X, F)$.

The following concept of gap function $g(x)$ associated with a general $\operatorname{VI}(X, F)$ will be utilized in this paper:

Definition 2 Given a problem $\operatorname{VI}(X, F)$, the gap function is defined to be

$$
g(x)=\max _{y \in X}\left\{F(x)^{T}(x-y)\right\} \text { for each } x \in X .
$$

Note that $g(x) \geq 0$ for each $x \in X$ and $g\left(x^{*}\right)=0$ if and only if $x^{*}$ is a solution to $\mathrm{VI}(X, F)$. In general, $g(x)$ may be nonconvex and nonsmooth. However, in our setting the value of $g(x)$ can be computed by some semi-infinite programming algorithms [S.-C. Fang and Tsao, 1997, Reemtsen and Ruckmann, 1998, S.-Y. Wu and Lin, 1998].

Also note the following definition for any $\varepsilon>0$ :

Definition 3 A point $\bar{x} \in X$ is called an $\varepsilon$-solution of the problem $\operatorname{VI}(X, F)$, if the gap function $g(\bar{x}) \leq \varepsilon$.

It is not difficult to see that, in this case, $F(\bar{x})^{T}(x-\bar{x}) \geq-\varepsilon$ for all $x \in X$.

\section{Discretization approach for $\operatorname{LSIVI}(X, F)$}

We first introduce a discretization approach for solving $\operatorname{LSIVI}(X, F)$. Since $T$ is a compact subset of $R$, there exists a positive-valued, strictly monotone decreasing function $\Delta$ from the natural numbers $N$ to the positive orthant $R_{+}$such that $\Delta(n) \rightarrow 0$ as $n \rightarrow \infty$ and an expansive sequence $\left\{T_{i}\right\}$ of finite subsets of $T$ with the property that for each $t \in T$, there exists an $n_{0} \in N$ such that, for $n \geq n_{0}$, there exists $t^{\prime} \in T_{n}$ with $\left\|t-t^{\prime}\right\| \leq \Delta(n)$. Using $T_{i}$, we define

$$
\bar{X}_{i}=\left\{x \in R^{n} \mid\langle u(t), x\rangle-\lambda(t) \leq 0 \text { for all } t \in T_{i}\right\}
$$

Note that $T_{i}$ is a finite subset of $T$ and $T_{i} \subset T_{i+1}$ for each $i$. Consequently, $X \subset \bar{X}_{i+1} \subset \bar{X}_{i}$.

Now consider the following variational inequality problem: $\operatorname{VI}\left(\bar{X}_{i}, F\right)$ : Find $x^{i} \in \bar{X}_{i}$ such that

$$
F\left(x^{i}\right)^{T}\left(x-x^{i}\right) \geq 0 \text { for all } x \in \bar{X}_{i} .
$$

The next result immediately follows:

Lemma 1 If $\bar{X}_{i}$ is bounded and $F$ is continuous on $\bar{X}_{i}$, then there exists a solution to VI $\left(\bar{X}_{i}, F\right)$.

Proof. It is not difficult to see that $\bar{X}_{i}$ is a closed convex set. If $\bar{X}_{i}$ is a bounded set, then $\bar{X}_{i}$ is a compact convex set. Since $F$ is continuous from $\bar{X}_{i}$ to $R^{n}$, the result follows from [Holmes, 1975].

In this case, we let $x^{i}$ be a solution of $\operatorname{VI}\left(\bar{X}_{i}, F\right)$. Next we show that there exists a subsequence of $\left\{x^{i}\right\}$ converging to a solution of $\operatorname{LSIVI}(X, F)$. 
Theorem 1 If there exists an $M>0$ such that $\left\|x^{i}\right\| \leq M$ for each $i$, then there exists a subsequence $\left\{x^{k_{i}}\right\}$ of $\left\{x^{i}\right\}$ converging to the solution of $\operatorname{LSIVI}(X, F)$.

Proof. Since $\left\|x^{i}\right\| \leq M$, there exists a subsequence $\left\{x^{k_{i}}\right\}$ converging to $x^{*}$. We claim that $\left\langle u(t), x^{*}\right\rangle-\lambda(t) \leq 0, \forall t \in T$. If not, there exists at least one $\bar{t} \in T$ such that $\left\langle u(\bar{t}), x^{*}\right\rangle-$ $\lambda(\bar{t})>0$. Hence there exists an $n_{1} \in\left\{k_{i}\right\}$ such that

$$
\left\langle u(\bar{t}), x^{n}\right\rangle-\lambda(\bar{t})>0 \text { for each } n \in\left\{k_{i}\right\} \text { and } n \geq n_{1} .
$$

From the definition of $T_{i}$, there exists $t_{i} \in T_{i}$ such that $t_{i} \rightarrow \bar{t}$ as $i \rightarrow \infty$. Consequently, there exists $n_{2}$ such that, for $i \geq n_{2}$,

$$
\left\langle u\left(t_{i}\right), x^{n}\right\rangle-\lambda\left(t_{i}\right) \geq 0 \text { for each } n \in\left\{k_{i}\right\} \text { and } n \geq n_{1} .
$$

Choose $n_{3} \geq \max \left\{n_{1}, n_{2}\right\}$, then

$$
\left\langle u\left(t_{n_{2}}\right), x^{n_{3}}\right\rangle-\lambda\left(t_{n_{2}}\right)>0 .
$$

Since $x^{n_{3}}$ is a solution of $\operatorname{VI}\left(\bar{X}_{n_{3}}, F\right)$,

$$
\left\langle u(t), x^{n_{3}}\right\rangle-\lambda(t) \leq 0 \text { for each } t \in T_{n_{3}} .
$$

For $t_{n_{2}} \in T_{n_{2}} \subseteq T_{n_{3}}$, we know $\left\langle u\left(t_{n_{2}}\right), x^{n_{3}}\right\rangle-\lambda\left(t_{n_{2}}\right) \leq 0$. This contradicts (16). Hence $\left\langle u(t), x^{*}\right\rangle-\lambda(t) \leq 0$ for any $t \in T$. Now we show that $F\left(x^{*}\right)^{T}\left(x-x^{*}\right) \geq 0$ for each $x \in X$. If not, we assume there exists at least one $\bar{x} \in X$ such that $F\left(x^{*}\right)^{T}\left(\bar{x}-x^{*}\right)<0$. Since $F$ is continuous, there exists $\bar{n} \in\left\{k_{i}\right\}$ such that

$$
F\left(x^{\bar{n}}\right)^{T}\left(\bar{x}-x^{\bar{n}}\right)<0 .
$$

On the other hand, since $\bar{x} \in X \subset \bar{X}_{\bar{n}}$, we have

$$
F\left(x^{\bar{n}}\right)^{T}\left(\bar{x}-x^{\bar{n}}\right) \geq 0 .
$$

This contradicts (18). Thus, for all $x \in X, F\left(x^{*}\right)^{T}\left(x-x^{*}\right) \geq 0$.

Note that although Theorem 1 assures the convergence of the discretization approach, the quality of solutions obtained by this approach depends on the choice of the expansive sequence $\left\{T_{i}\right\}$. Usually finer discretization results in better approximation, however it is hard to provide a quantitative statement.

\section{Inexact cutting plane approach for $\operatorname{LSIVI}(X, F)$}

In this section, we present an analytic center based inexact cutting plane method for solving $\operatorname{LSIVI}(X, F)$.

Given $t_{1}, \ldots, t_{m}$ be $m$ points in $T$, define

$$
T_{1}=\left\{t_{1}, \ldots, t_{m}\right\} \subset T
$$


and

$$
X_{1}=\left\{x \in R^{n} \mid\langle u(t), x\rangle-\lambda(t) \leq 0 \text { for all } t \in T_{1}\right\} .
$$

Since $u: T_{1} \subset T \rightarrow R^{n}$, we can define an $m \times n$ matrix $A$ with $A_{i}=u\left(t_{i}\right)$ being its $i$ th row for $i=1, \ldots, m$. Similarly, since $\lambda: T_{1} \rightarrow R$, we define $b$ to be an $m$ vector with $b_{i}=\lambda\left(t_{i}\right)$ being the $i$ th component of $b$ for $i=1, \ldots, m$. Then $X_{1}$ can be rewritten as a polyhedral set $\left\{x \in R^{n} \mid A x \leq b\right\}$.

For a variational inequality problem like $\mathrm{VI}\left(X_{1}, F\right)$, J.-L. Goffin and Zhu presented an analytic center based cutting plane method to solve it [1997]. They showed that under some technical conditions such as $F$ being pseudomonotone-plus and Lipschitz continuous on $X_{1}$, and the inequalities $0 \leq x \leq e$ (where $e$ is the vector of all 1's) are included in the system $A x \leq b$, their algorithm either terminates with an exact solution of $\operatorname{VI}\left(X_{1}, F\right)$ in a finite number of iterations, or generates an infinite sequence $\left\{x^{k}\right\}$ that has a subsequence converging to a solution of $\mathrm{VI}\left(X_{1}, F\right)$. In the latter case, when $k$ is sufficiently large, $x^{k}$ becomes an $\varepsilon$ solution to $\mathrm{VI}\left(X_{1}, F\right)$, for any given $\varepsilon>0$.

Following this idea, we present an inexact cutting plane algorithm for solving $\operatorname{LSIVI}(X, F)$.

Algorithm 4.1 Given $\bar{\varepsilon}>0, \bar{\delta} \in(0,1), T_{1}=\left\{t_{1}, \ldots t_{m}\right\}$, and $X_{1}=\left\{x \in R^{n} \mid\langle u(t), x\rangle-\right.$ $\lambda(t) \leq 0$ for all $\left.t \in T_{1}\right\}$, set $k=1$.

Step 1. Find an $\bar{\varepsilon}(1-\bar{\delta})^{k}$-solution $x^{k}$ to $\operatorname{VI}\left(X_{k}, F\right)$.

Step 2. Let $t_{m+k}$ be a maximizer of $\left\langle u(t), x^{k}\right\rangle-\lambda(t)$ over $T$.

If $\left\langle u\left(t_{m+k}\right), x^{k}\right\rangle-\lambda\left(t_{m+k}\right)>0$, then go to Step 3.

Otherwise, if $x^{k}$ is an exact solution to $\operatorname{VI}\left(X_{k}, F\right)$, then stop and output $x^{k}$.

Otherwise, set $X_{k+1} \leftarrow X_{k}, k \leftarrow k+1$, then go to Step 1 .

Step 3. Let $T_{k+1}=T_{k} \cup\left\{t_{m+k}\right\}$ and $X_{k+1}=\left\{x \in R^{n} \mid\langle u(t), x\rangle-\lambda(t) \leq 0\right.$ for all $t \in$ $\left.T_{k+1}\right\}$. Update $k \leftarrow k+1$, go to Step 1 .

Note that in Step 1, only an inexact solution to a subprogram $\operatorname{VI}\left(X_{k}, F\right)$ is needed at each iteration. This task can be carried out by using the analytic center cutting plane method proposed in [J.-L. Goffin and Zhu, 1997]. Also in Step 3, only one cutting plane is added in each time. Hence we say this is an analytic center based inexact cutting plane approach. Next we show a convergence result.

Theorem 2 Algorithm 4.1 either (i) terminates with a solution to $\operatorname{LSIVI}(X, F)$ in a finite number of iterations at Step 2, or (ii) generates an infinite sequence that has a subsequence converging to a solution to $\operatorname{LSIVI}(X, F)$.

Proof. (i) Suppose that the Algorithm 4.1 terminates in $\bar{n}$ iterations. To meet the stopping criterion in Step 2, we know

$$
\left\langle u(t), x^{\bar{n}}\right\rangle-\lambda(t) \leq 0 \text { for all } t \in T
$$

and

$$
F\left(x^{\bar{n}}\right)^{T}\left(x-x^{\bar{n}}\right) \geq 0 \text { for all } x \in X_{\bar{n}} .
$$


Since $X \subseteq X_{\bar{n}}$, from (23), we have

$$
F\left(x^{\bar{n}}\right)^{T}\left(x-x^{\bar{n}}\right) \geq 0 \text { for all } x \in X .
$$

From (22), we know $x^{\bar{n}} \in X$. Thus $x^{\bar{n}}$ is a solution to $\operatorname{LSIVI}(X, F)$.

(ii) Assume that Algorithm 4.1 does not terminate in a finite number of iterations. Consider the sequence $\left\{x^{n_{i}}\right\}$, where each index $n_{i}$ is obtained from Step 3 only. Like in [J.-L. Goffin and Zhu, 1997], the linear inequality system $X_{1}=\left\{x \in R^{n} \mid A x \leq b\right\}$ is assumed to include the inequalities $0 \leq x \leq e$. Hence it is a compact set.

Since $x^{n_{i}} \in X_{n_{i}} \subset X_{1}$ and $t_{m+n_{i}} \in T$, there exist subsequences $\left\{x^{n_{k_{i}}}\right\}$ of $\left\{x^{n_{i}}\right\}$ converging to $x^{*} \in X_{1}$, and $\left\{t_{m+n_{k_{i}}}\right\}$ of $\left\{t_{m+n_{i}}\right\}$ converging to $t_{*} \in T$. We claim that

$$
\left\langle u\left(t_{m+n_{i}}\right), x^{*}\right\rangle-\lambda\left(t_{m+n_{i}}\right) \leq 0 \text { for } i=1,2, \cdots
$$

If not, then there exists a $p \in N$ such that

$$
\left\langle u\left(t_{m+n_{p}}\right), x^{*}\right\rangle-\lambda\left(t_{m+n_{p}}\right)>0
$$

Since $\left\{x^{n_{k_{i}}}\right\} \rightarrow x^{*}$, there exists a sufficiently large $q \in N$ such that $n_{q}>m+n_{p}$ and

$$
\left\langle u\left(t_{m+n_{p}}\right), x^{n_{q}}\right\rangle-\lambda\left(t_{m+n_{p}}\right)>0
$$

Moreover, $x^{n_{q}}$ is an $\bar{\varepsilon}(1-\bar{\delta})^{n_{q}}$ solution of $\operatorname{VI}\left(X_{n_{q}}, F\right)$, we know

$$
\left\langle u\left(t_{m+n_{p}}\right), x^{n_{q}}\right\rangle-\lambda\left(t_{m+n_{p}}\right) \leq 0 .
$$

Notice that (28) contradicts (27), we know (25) holds.

Let $\bar{t}$ be a maximizer of the function $\left\langle u(t), x^{*}\right\rangle-\lambda(t)$ over T. Since $t_{m+n_{k_{i}}}$ is a maximizer of $\left\langle u(t), x^{n_{k_{i}}}\right\rangle-\lambda(t)$ over $T$,

$$
\left\langle u(\bar{t}), x^{n_{k_{i}}}\right\rangle-\lambda(\bar{t}) \leq\left\langle u\left(t_{m+n_{k_{i}}}\right), x^{n_{k_{i}}}\right\rangle-\lambda\left(t_{m+n_{k_{i}}}\right) .
$$

Let $i \rightarrow \infty$ in (29), we see

$$
\left\langle u(\bar{t}), x^{*}\right\rangle-\lambda(\bar{t}) \leq\left\langle u\left(t_{*}\right), x^{*}\right\rangle-\lambda\left(t_{*}\right) .
$$

It follows from (25) that

$$
\left\langle u\left(t_{*}\right), x^{*}\right\rangle-\lambda\left(t_{*}\right) \leq 0
$$

Therefore,

$$
\left\langle u(\bar{t}), x^{*}\right\rangle-\lambda(\bar{t}) \leq\left\langle u\left(t_{*}\right), x^{*}\right\rangle-\lambda\left(t_{*}\right) \leq 0,
$$

and hence $x^{*} \in X$.

Now we show that

$$
F\left(x^{*}\right)^{T}\left(x-x^{*}\right) \leq 0 \text { for all } x \in X .
$$

For each $i$, since $x^{n_{k_{i}}}$ is an $\bar{\varepsilon}(1-\bar{\delta})^{n_{k_{i}}}$ solution of $\operatorname{VI}\left(X_{n_{k_{i}}}, F\right)$, we know

$$
F\left(x^{n_{k_{i}}}\right)^{T}\left(x-x^{n_{k_{i}}}\right) \geq-\bar{\varepsilon}(1-\bar{\delta})^{n_{k_{i}}} \text { for all } x \in X \subset X_{n_{k_{i}}} .
$$


When $i \rightarrow \infty$, we have

$$
F\left(x^{*}\right)^{T}\left(x-x^{*}\right) \geq 0 \text { for all } x \in X .
$$

Thus $x^{*}$ is a solution to $\operatorname{LSIVI}(X, F)$.

Suppose that Algorithm 4.1 does not terminate in a finite number of iterations, but after $s$-th iteration we have a $\bar{\varepsilon}(1-\bar{\delta})^{s}$-solution $x^{s}$ for $\mathrm{VI}\left(X_{s}, F\right)$. Assume that there exists a small $\delta^{\prime}>0$ such that

$$
\left\langle u(t), x^{s}\right\rangle-\lambda(t) \leq \delta^{\prime} \text { for all } t \in T \text {. }
$$

From Theorem $2, x^{s}$ can be viewed as an approximate solution of $\operatorname{LSIVI}(X, F)$, if $\delta>0$ is sufficiently small. An interesting, yet important, question is "how good such an approximate solution can be?" The following theorem addresses this issue under some technical conditions.

Theorem 3 If there exists an $\bar{x} \in R^{n}$ such that (i) $\langle u(t), \bar{x}\rangle \geq 1$ for all $t \in T$ and (ii)

$F(x)^{T} \bar{x} \geq 1$ for all $x \in X$, then $x^{s}-\delta^{*} \bar{x}$, where $\delta^{*}=\max \left\{\delta^{\prime}, \bar{\varepsilon}(1-\bar{\delta})^{s}\right\}$, is a solution of $\operatorname{LSIVI}(X, F)$.

Proof. Since for every $t \in T$

$$
\left\langle u(t), x^{s}\right\rangle-\lambda(t) \leq \delta^{\prime}
$$

and

$$
\left\langle u(t), \delta^{*} \bar{x}\right\rangle \geq \delta^{*}
$$

we know

$$
\left\langle u(t), x^{s}-\delta^{*} \bar{x}\right\rangle-\lambda(t)=\left\langle u(t), x^{s}\right\rangle-\lambda(t)-\left\langle u(t), \delta^{*} \bar{x}\right\rangle \leq \delta^{\prime}-\delta^{*} \leq 0 .
$$

Consequently, $x^{s}-\delta^{*} \bar{x} \in X$.

Since for every $x \in X$

$$
F(x)^{T}\left(x-x^{s}\right) \geq-\bar{\varepsilon}(1-\bar{\delta})^{s}
$$

and

$$
F(x)^{T}\left(\delta^{*} \bar{x}\right) \geq \delta^{*}
$$

we know

$$
\begin{aligned}
F(x)^{T}\left(x-\left(x^{s}-\delta^{*} \bar{x}\right)\right) & =F(x)^{T}\left(x-x^{s}\right)+F(x)^{T}\left(\delta^{*} \bar{x}\right) \\
& \geq-\bar{\varepsilon}(1-\bar{\delta})^{s}+\delta^{*} \geq 0 .
\end{aligned}
$$

Therefore, $x^{s}-\delta^{*} \bar{x}$ is a solution of $\operatorname{DLSIVI}(X, F)$. From Proposition 2, we know $x^{s}-\delta^{*} \bar{x}$ is also a solution of $\operatorname{LSIVI}(X, F)$.

Notice that the condition assumed in Theorem 3 is a technical condition that may be difficult to check in general. But when it is satisfied, we know how close $x^{s}$ can be a solution to $\operatorname{LSIVI}(X, F)$. In some special cases, this technical condition can be verified easily. For example, if $u(t)=\left(u_{1}(t), \ldots, u_{n}(t)\right)>0$ for $t \in T$ and $F(x)=\left(F_{1}(x), \ldots, F_{n}(x)\right)>0$ for $x \in X$, then the condition is clearly satisfied. Also notice that when the analytic center cutting plane algorithm of [J.-L. Goffin and Zhu, 1997] is used, under the assumption that $F$ is pseudo co-coercive and Lipschitz continuous on $X_{i}$, an approximation solution $x^{i}$ to $\operatorname{VI}\left(X_{i}, F\right)$ can be found in polynomial time. Therefore, in this case, an $\varepsilon$-solution of $\operatorname{LSIVI}(X, F)$ can be achieved in polynomial time. 


\section{Numerical Examples}

In this section we provide numerical examples to illustrate the potentials of discretization and inexact cutting plane approaches. We have implemented both approaches in MATLAB on a $1000 \mathrm{MHz}$ Pentium III personal computer running Linux. Recall that for both approaches, a finite dimensional variational inequality subproblem needs to be solved. For this purpose, we have implemented the method proposed by [J.-L. Goffin and Zhu, 1997]. To compute the approximate analytic center in Goffin's method, we have used Newton's linear approximation along with a dual scaling procedure [J.-L. Goffin and Ye, 1996]. We have assumed that an exact solution is found at this step, if the solution is within the accuracy of $1.0 \mathrm{e}-8$. The following examples are studied in the sequel:

Example $1 n=7, T=[0,1]$, and

$$
\begin{gathered}
X=\left\{x \in R^{7} \mid \sum_{j=1}^{7} t^{j-1} x_{j} \leq \sum_{l=1}^{4} t^{2 l}+1, t \in T \text { and } 0 \leq x_{j} \leq 1, j=1, \cdots, 7\right\} \\
F=\left(F_{1}, \cdots, F_{7}\right) \text { with } F_{j}=x_{j}-\frac{1}{\sqrt{x_{j}}}, j=1, \cdots, 7
\end{gathered}
$$

Example $2 n=7, T=[0,1]$, and

$$
\begin{gathered}
X=\left\{x \in R^{7} \mid \sum_{j=1}^{7} t^{j-1} x_{j} \leq 4 t^{5}+1, t \in T \text { and } 0 \leq x_{j} \leq 1, j=1, \cdots, 7\right\}, \\
F=\left(F_{1}, \cdots, F_{7}\right) \text { with } F_{j}=1+3 x_{j}-\frac{1}{x_{j}^{2}}, j=1, \cdots, 7
\end{gathered}
$$

Example $3 n=7, T=[0,1]$, and

$$
\begin{gathered}
X=\left\{x \in R^{7} \mid \sum_{j=1}^{7} t^{j-1} x_{j} \leq 3 t^{5}+2 t^{2}+\frac{1}{3}, t \in T \text { and } 0 \leq x_{j} \leq 1, j=1, \cdots, 7\right\}, \\
F=\left(F_{1}, \cdots, F_{7}\right) \text { with } F_{j}=\sqrt{x_{j}}-\frac{1}{x_{j}^{2}}, j=1, \cdots, 7 .
\end{gathered}
$$

Notice that, for the above examples the "interior point" assumption (5) is satisfied when we set $\hat{x}$ to $0.1 e$ ( $e$ is the vector of all 1's). To apply Goffin's method, the inequalities $0 \leq x \leq e$ are included in the system of linear inequalities. Hence, the set $X$ has become nonempty, convex and compact for each example.

First we have solved the examples using the inexact cutting plane approach (Algorithm 4.1). For all the examples, $\bar{\varepsilon}$ and $\bar{\delta}$ are set to 0.1 and 0.5 , respectively. Also, the initial set $T_{1}$ is taken as $\{0.0,1.0\}$. The second column of Table 1 gives the number of iterations for finding the solution $x^{*}$. The third column shows $x^{*}$ and the final partition $T_{k}$ reported by the algorithm. Recall that in Step 2 of Algorithm 4.1 if $x^{k}$ is not an exact solution, at the next iteration the algorithm moves back to Step 1 without adding a new cutting plane. Therefore, the number of iterations in the second column are higher than the number of partitions in the third column. 


\begin{tabular}{|c|c|c|}
\hline Ex. & $k$ & $x^{*}$ and $T_{k}$ \\
\hline \hline \multirow{2}{*}{1} & \multirow{2}{*}{12} & $\begin{array}{c}x^{*}=(0.4999,0.5662,0.6297,0.6853,0.7344,0.7769,0.8123)^{T} \\
T_{12}=\{0.0,0.8136,0.8270,0.8287,0.8299,0.8335,1.0\}\end{array}$ \\
\hline 2 & \multirow{2}{*}{11} & $\begin{array}{c}x^{*}=(0.5080,0.5361,0.5564,0.5699,0.5797,0.5862,0.5902)^{T} \\
T_{11}=\{0.0,0.6468,0.6482,0.6733,1.0\}\end{array}$ \\
\hline 3 & \multirow{2}{*}{15} & $\begin{array}{l}x^{*}=(0.2764,0.4799,0.7236,0.8933,0.9662,0.9899,0.9968)^{T} \\
T_{15}=\{0.0,0.2890,0.2903,0.2906,0.2914,0.3074,0.6045,1.0\}\end{array}$ \\
\hline
\end{tabular}

Table 1: Solutions using the inexact cutting plane approach.

\begin{tabular}{|c|c|l|}
\hline Ex. & NOP & $x^{*}$ \\
\hline \hline & 10 & $x^{*}=(0.4782,0.5594,0.6316,0.6986,0.7537,0.8027,0.8399)$ \\
1 & 20 & $x^{*}=(0.5058,0.5715,0.6301,0.6839,0.7305,0.7712,0.8055)$ \\
& 40 & $x^{*}=(0.4958,0.5659,0.6306,0.6867,0.7364,0.7793,0.8158)$ \\
& 80 & $x^{*}=(0.4987,0.5663,0.6303,0.6856,0.7340,0.7781,0.8128)$ \\
\hline \multirow{4}{*}{2} & 10 & $x^{*}=(0.5100,0.5413,0.5623,0.5763,0.5845,0.5899,0.5933)$ \\
& 20 & $x^{*}=(0.5084,0.5360,0.5559,0.5699,0.5795,0.5858,0.5901)$ \\
& 40 & $x^{*}=(0.5084,0.5359,0.5560,0.5698,0.5796,0.5857,0.5901)$ \\
& 80 & $x^{*}=(0.5082,0.5360,0.5560,0.5700,0.5797,0.5859,0.5901)$ \\
\hline \multirow{4}{*}{3} & 10 & $x^{*}=(0.2785,0.4767,0.7145,0.8860,0.9603,0.9874,0.9963)$ \\
& 20 & $x^{*}=(0.2785,0.4766,0.7143,0.8852,0.9623,0.9880,0.9970)$ \\
& 40 & $x^{*}=(0.2769,0.4793,0.7214,0.8914,0.9645,0.9892,0.9969)$ \\
& 80 & $x^{*}=(0.2760,0.4802,0.7246,0.8930,0.9664,0.9902,0.9970)$ \\
\hline
\end{tabular}

Table 2: Solutions using the discretization approach.

Table 2 shows the solutions found by the discretization approach. The set $T$ has been divided into equally spaced partitions, and to analyze the effect of finer discretization, the number of partitions (NOP) has been varied from 10 to 80 . The second column gives the NOP required to achieve $x^{*}$ in the last column.

From Table 1, we see that the inexact cutting plane approach converges to the solutions of the three examples with only 6,4 , and 7 partitions, respectively. In the meanwhile, the third column in Table 2 shows that the discretization approach converges to the corresponding solutions at the expense of more partitions. Also note that, finer partitioning for the discretization approach leads to solutions that are closer to the solutions confirmed by the inexact cutting plane approach.

\section{Conclusion}

In this paper, we have studied a special class of variational inequalities over a domain defined by infinitely many linear inequalities. A discretization approach for solving such problems 
is introduced with a convergence proof. We also propose an "inexact" cutting plane method based on analytic centers. A convergence proof and several numerical examples are included. Under proper conditions, we can examine the quality of solutions obtained. In particular, an $\varepsilon$-optimal solution can be generated by the proposed algorithm in polynomial time.

\section{References}

J.-L. Goffin and J.-P. Vial. Convex nondifferentiable optimization: a survey focused on the analytic center cutting plane method. Technical report, Department of Management Studies, University of Geneva, Switzerland, 1999.

P. T. Harker and J.-S. Pang. Finite-dimensional variational inequality and nonlinear complementarity problems: a survey of theory, algorithms and applications. Mathematical Programming, 48:161-220, 1990.

R. B. Holmes. Geometric Functional Analysis and Its Applications. Springer-Verlag, New York, 1975.

P. Marcotte J.-L. Goffin and D. Zhu. An analytic center cutting plane method for pseudomonotone variational inequalities. Operations Research Letters, 20:1-6, 1997.

Z. Luo J.-L. Goffin and Y. Ye. Complexity analysis of an interior cutting plane method for convex feasibility problems. SIAM J. Optimization, 6:638-652, 1996.

Z.-Q. Luo J.-L. Goffin and Y. Ye. On the complexity of a column generation algorithm for convex or quasiconvex feasibility problems. In Hearn W. W. Hager, D. W and P. M. Pardalos, editors, Large Scale Optimization: State of The Art. Kluwer Academic Publishers, 1993.

Z.-Q. Luo and J. Sun. An analytic center based column generation algorithm for convex quadratic feasibility problems. SIAM J. Optimization, 9:217-235, 1999.

Z.-Q. Luo and J. Sun. A polynomial cutting surfaces algorithm for the convex feasibility problem defined by self-concordant inequalities. Computational Optimization and Applications, 15:167-191, 2000.

H.-J. Lüthi and B. Bueler. An analytical center quadratic cut method for strongly monotone variational inequality problems. SIAM J. Optimization, 10:415-426, 2000.

T. L. Magnanti and G. Perakis. A unifying geometric solution framework and complexity analysis for variational inequalities. Mathematical Programming, 71:327-351, 1995.

P. Marcotte and D. Zhu. A cutting plane method for solving quasimonotone variational inequalities. Computational Optimization and Applications, 20:317-324, 2001.

R. Reemtsen and J.-J. Ruckmann, editors. Semi-Infinite Programming. Kluwer Academic Publishers, The Netherlands, 1998. 
J. R. Rajasekera S.-C. Fang and H.-S. J. Tsao. Entropy Optimization and Mathematical Programming. Kluwer Academic Publishers, Boston, MA, 1997.

S.-Y. Wu S.-C. Fang and J. Sun. An analytic center cutting plane method for solving semiinfinite variational inequality problems. Submitted to Journal of Global Optimization, 2002.

S.-C. Fang S.-Y. Wu and C.-J. Lin. Relaxed cutting plane method for solving linear semiinfinite programming problems. Journal of Optimization Theory and Applications, 99:759779, 1998. 


\section{Publications in the Report Series Research* in Management}

ERIM Research Program: "Business Processes, Logistics and Information Systems"

\section{2}

The importance of sociality for understanding knowledge sharing processes in organizational contexts Niels-Ingvar Boer, Peter J. van Baalen \& Kuldeep Kumar ERS-2002-05-LIS

Crew Rostering for the High Speed Train

Ramon M. Lentink, Michiel A. Odijk \& Erwin van Rijn

ERS-2002-07-LIS

Equivalent Results in Minimax Theory

J.B.G. Frenk, G. Kassay \& J. Kolumbán

ERS-2002-08-LIS

An Introduction to Paradigm

Saskia C. van der Made-Potuijt \& Arie de Bruin

ERS-2002-09-LIS

Airline Revenue Management: An Overview of OR Techniques 1982-2001

Kevin Pak \& Nanda Piersma

ERS-2002-12-LIS

Quick Response Practices at the Warehouse of Ankor

R. Dekker, M.B.M. de Koster, H. Van Kalleveen \& K.J. Roodbergen

ERS-2002-19-LIS

Harnessing Intellectual Resources in a Collaborative Context to create value

Sajda Qureshi, Vlatka Hlupic, Gert-Jan de Vreede, Robert O. Briggs \& Jay Nunamaker

ERS-2002-28-LIS

Version Spaces and Generalized Monotone Boolean Functions

Jan C. Bioch \& Toshihide Ibaraki

ERS-2002-34-LIS

Periodic Review, Push Inventory Policies for Remanufacturing

B. Mahadevan, David F. Pyke, Moritz Fleischman

ERS-2002-35-LIS

Modular Decomposition of Boolean Functions

Jan C. Bioch

ERS-2002-37-LIS

Classification Trees for Problems with Monotonicity Constraints

R. Potharst \& A.J. Feelders

ERS-2002-45-LIS

A complete overview of the ERIM Report Series Research in Management: http://www.ers.erim.eur.nl

ERIM Research Programs:

LIS Business Processes, Logistics and Information Systems

ORG Organizing for Performance

MKT Marketing

F\&A Finance and Accounting

STR Strategy and Entrepreneurship 
Allocation of Railway Rolling Stock for Passenger Trains

Erwin Abbink, Bianca van den Berg, Leo Kroon \& Marc Salomon

ERS-2002-47-LIS

Monotone Decision Trees and Noisy Data

Jan C. Bioch and Viara Popova

ERS-2002-53-LIS

Business Modeling Framework For Personalization In Mobile Business Services: a Case and Sociological Analysis L-F Pau, Jeroen Dits

ERS-2002-56-LIS

Polynomial time algorithms for some multi-level lot-sizing problems with production capacities

Stan van Hoesel, H. Edwin Romeijn, Dolores Romero Morales, Albert P.M. Wagelmans

ERS-2002-59-LIS

A Note on Ending Inventory Valuation in Multiperiod Production Scheduling

Wilco van den Heuvel, Alfred P.M. Wagelmans

ERS-2002-63-LIS

Determining The Optimal Order Picking Batch Size In Single Aisle Warehouses

Tho Le-Duc and René B.M. de Koster

ERS-2002-64-LIS

Solving Variational Inequalities Defined on A Domain with Infinitely Many Linear Constraints

Shu-Cherng Fang, Soonyi Wu, Ş. İlker Birbil

ERS-2002-70-LIS

Entropic Regularization Approach for Mathematical Programs with Equilibrium Constraints

Ş. İlker Birbil, Shu-Cherng Fang, Jiye Han

ERS-2002-71-LIS

On the Finite Termination of An Entropy Function Based Smoothing Newton Method for Vertical Linear Complementarity Problems

Shu-Cherng Fang, Jiye Han, Zhenghai Huang, Ş. İlker Birbil

ERS-2002-72-LIS

The Role Of Product Quality Information, Market State Information And Transaction Costs In Electronic Auctions Otto Koppius and Eric van Heck

ERS-2002-73-LIS 